\section{Pain management after lung surgery}

\author{
Maria Frödin, ${ }^{1}$ \\ Margareta Warrén Stomberg ${ }^{2}$
}

1Sahlgrenska University

Hospital/Mölndal, Mölndal; ${ }^{2}$ University of

Gothenburg/The Sahlgrenska Academy,

Institute of Health and Care Sciences,

Gothenburg, Sweden

\section{Abstract}

Pain management is an integral challenge in nursing and includes the responsibility of managing patients' pain, evaluating pain therapy and ensuring the quality of care. The aims of this study were to explore patients' experiences of pain after lung surgery and evaluate their satisfaction with the postoperative pain management. A descriptive design was used which studied 51 participants undergoing lung surgery. The incidence of moderate postoperative pain varied from 36 $58 \%$ among the participants and severe pain from 11-26\%, during their hospital stay. Thirty-nine percent had more pain than expected. After three months, 20\% experienced moderate pain and $4 \%$ experienced severe pain, while after six months, $16 \%$ experienced moderate pain. The desired quality of care goal was not fully achieved. We conclude that a large number of patients experienced moderate and severe postoperative pain and more than one third had more pain than expected. However, $88 \%$ were satisfied with the pain management. The findings confirm the severity of pain experienced after lung surgery and facilitate the apparent need for the continued improvement of postoperative pain management following this procedure.

\section{Introduction}

Patients undergoing lung surgery run a risk of suffering from postoperative pain and some may develop chronic pain. ${ }^{1,2}$ Chronic pain has been found in $21-61 \%$ of patients after lung surgery. ${ }^{3}$ Furthermore, studies have found that lung surgery may cause long-term negative effects for the patients, in terms of suffering, reduced quality of life and an ultimately increased cost to society. ${ }^{4,5}$ According to the national guidelines developed by the Swedish Society of Anaesthesia and Intensive Care, pain should be treated preventively, using an individual approach that involves patients in their pain management. As part of quality care assurance, the overall purpose of this study was to explore patients' experience of pain after lung surgery and, from their perspective, evaluate the quality as well as satisfaction regarding the postoperative pain management.

\section{Surgical procedure and pain managements}

In lung surgery, the surgical technique is either an open one via thoracotomy or video-assisted thoracic surgery (VATS) using a minimally invasive endoscopic procedure. Thoracic epidural analgesia (TEA), has been the golden standard of pain management after lung surgery via thoracotomy. ${ }^{6} 7$ However, paravertebral block, (PVB), has been shown to provide comparable pain relief to TEA, but with fewer side effects. ${ }^{7,8}$ If TEA or PVB are not suitable, intercostal nerve blocks, bolus and continuous infusion, combined with IV analgesic, are recommended. Complementary medications as part of multimodal analgesia, such as paracetamol, weak opioids and non-steroidal anti-inflammatory drugs, are also recommended. ${ }^{9,7}$

\section{Acute and prolonged}

\section{post-surgical pain}

A prolonged and high impulse flow in a nerve can lead to a persistent up-regulation of activity, from the spine and inwardly. This refers to be wind-up, indicating the pain has been sustaining and strengthened as well. A central sensitization may also appear with increased sensitivity in the receptors around the injured area, which leads to a conversion of pressure and touch signals to nociceptive signals, which in turn indicate pain. Offensive and multimodal pain management minimizes the risk of secondary hyperalgesia and the wind-up phenomena and therefore the development of chronic pain. ${ }^{6}$ Steegers et al. ${ }^{10}$ found that up to half of the patients with chronic pain did not have a neuropathic pain component, which suggests other causes for the chronic pain, such as a visceral pain component. Furthermore, a number of predisposing factors for the development of chronic pain and disorder after surgery have been identified: preoperative pain and anxiety, female sex, type of surgery, ${ }^{1,3}$ severe pain during the first few postoperative days, depression and anxiety, young age,,$^{3,11}$ and the length of surgery. ${ }^{1,10,12}$

\section{Nursing perspective}

\section{in postoperative pain management}

Pain management for surgical patients is integral to nursing. The knowledge and attitude of nurses towards pain management will affect patients' postoperative pain management (POPM). Educational programs have proven to increase nurses' knowledge about POPM and their competence of managing patient prob-
Correspondence: Margareta Warrén Stomberg, University of Gothenburg/The Sahlgrenska Academy, Institute of Health and Care Sciences, PO Box 457, SE 40530 Gothenburg, Sweden. Tel.: +46.31.7866026 - Fax: +46.31.7866050.

E-mail: margareta.warren.stomberg@gu.se

Key words: postoperative pain management, lung surgery, satisfaction, expectation.

Contributions: MWS, study design, manuscript analysis, preparation and final editing; MF, design, data collection, data analysis and manuscript preparation. Both authors read and approved the final manuscript, as well as the revision.

Conflict of interests: the authors declare no potential conflict of interests.

Received for publication: 11 March 2014.

Revision received: 29 May 2014.

Accepted for publication: 30 May 2014.

This work is licensed under a Creative Commons Attribution NonCommercial 3.0 License (CC BYNC 3.0).

(C) Copyright M. Frödin and M. Warrén Stomberg, 2014

Licensee PAGEPress, Italy

Nursing Reports 2014; 4:3225

doi:10.4081/nursrep.2014.3225

lems arising from POPM, ${ }^{13}$ which also affect patients' experiences of and satisfaction with the nursing care provided.

Wilson ${ }^{14}$ showed that specialist nurses have a more comprehensive knowledge base in general pain management, compared to general nurses. It seems this knowledge base was influenced by the specialist nurses' education rather than their experience of years working in the nursing profession. Other studies ${ }^{11,15}$ have found a discrepancy between patients' and nurses' pain reports. The result indicated that nurses overestimated mild pain and underestimated severe pain. ${ }^{11}$ Dihle et al. ${ }^{15}$ observed nurses' actions and after interviewing them found a discrepancy between their words and actions in the postoperative pain management, which demonstrated that the nurses had theoretical knowledge about POPM, but did not always use it in the clinical setting. Nurses with specialist training are closely involved in the peri-operative pain management. They have a responsibility to effectively manage patients' pain, offer information and education to patients about different options regarding pain management, as well as ensure that patients' pain problems are recorded and evaluated. ${ }^{16}$

The aims of this study were to explore patients' experience of pain after lung surgery and from the patients' perspective, evaluate 
the quality of and satisfaction with the postoperative pain management.

\section{Materials and Methods}

This was a descriptive study which consecutively included participants from the waiting list for lung surgery, from May to December 2011, at a department of vascular and cardiothoracic surgery, at a university hospital in southwest Sweden.

\section{Sample}

In this study 52 patients were consecutively invited to participate. Inclusion criteria were adult patients (aged 18 or above) undergoing elective lung surgery and able to read and understand the questions in Swedish. Patients were excluded if they were diagnosed with mental disorders, experienced pain for more than three months before surgery and/or had a regular intake of analgesic(s) at least three days/week, required emergency care, and/or had lung surgery previously. Ten patients from the list of scheduled surgery were not invited to participate due to one of the above exclusion criteria. According to current routine practice of the surgical unit for lung surgery, the patients were supposed to have about five days of hospital stay. On the postoperative day (POD) 3, 4 and 5, four, 11 and 11 patients were discharged respectively. At the third and sixth month of data collection, a total of two patients were withdrawn due to critical illness (i.e., the remaining $n=49$ ).

\section{Instruments used to measure pain and quality of pain management}

The visual analogue scale (VAS) was used to assess patients' experience of pain after the surgical procedure. The VAS score was assessed by the patients themselves and, in the study, the recommended VAS score target was VAS $<40 \mathrm{~mm}$.

\section{Strategic and clinical quality indicators in postoperative pain management}

The validated Swedish version of the strategic and clinical quality indicators in postoperative pain management (SCQIPP) questionnaire with 14 items was adopted to assess the quality of postoperative pain management. The items mainly covered a few important aspects, including preoperative education about pain management and treatment offered after surgery, when the patient should be asked about his/her pain at movement, trusting the patient about his/her pain perception, treating the pain adequately, and cooperative approach to pain treatment. The items in the question- naire, where higher values indicated higher quality of pain management, scored on a 5point scale from $1=$ strongly disagree to $5=$ strongly agree. It was suggested that a mean score of $>4.5$ indicated a high quality of care, ${ }^{17}$ and $<4$ a low quality of care. ${ }^{18,19}$ To achieve a high quality of care in the postoperative pain management, the desired total mean score must be $\geq 63 / 70$ (14 items $\times 4.5$ ). The complementary questions in the instrument were: Has the patient had more pain than expected? rated with Yes/No. How satisfied/dissatisfied was the patient with the overall pain management? scored on a 5-point Likert scale ranging from $1=$ very dissatisfied to $5=$ very satisfied. ${ }^{17}$

\section{Hospital anxiety and depression scale}

The hospital anxiety and depression (HAD) scale measured anxiety and depression and each item was rated between $0-3$. The total score ranged from $0-21$ in the each of the two subscales (HAD-A and HAD-D). HAD-A and/or HAD-D $\leq 7$ would indicate no or very mild anxiety or depression, whereas their scores $\geq 8-10$ could indicate a possible anxiety or depression and scores $\geq 11$ could indicate a probable anxiety or depression. ${ }^{20}$

\section{Pain management routines}

According to the local guidelines in Sweden, TEA is the first choice of postoperative pain management. A TEA catheter is inserted and tested either the day prior to surgery or on the same day and activated at anesthesia induction. A bolus dose of sufentanil and bupivacain is administered before it is activated. TEA continues until the chest tube is removed. The epidural infusion is a combination of bupivacain $1 \mathrm{mg} / \mathrm{mL}$, fentanyl $2 \mu \mathrm{g} / \mathrm{mL}$ and adrenalin $2 \mu \mathrm{g} / \mathrm{mL}$. VAS $<40 \mathrm{~mm}$ has to be achieved before the patient returns to the general ward. Each patient has a protocol for the prescribed analgesic drugs, rates, as well as a checklist for basic and specific controls. This is documented on at least every nursing shift, i.e., three times per day.

IV analgesic is given to those patients for whom TEA is unsuitable. The drug used is morphine $1 \mathrm{mg} / \mathrm{mL}$ as infusion and bolus, pro re nata. The patients with IV analgesic also received intercostal nerve block, as a single injection at the end of surgery. As additional oral analgesic, oxycodone was given twice a day and as rescue analgesic.

\section{Procedure}

After informed consent the participants were asked about preoperative pain and regular intake of analgesia, as well as other patient characteristics. A regular intake of analgesia was defined as more than three times per week. The patients included in the study com- pleted the Swedish version of the HAD scale during their preoperative ward visit. They also completed the HAD scale on the day of discharge or latest on POD 5. In order to assess the quality of postoperative pain management, the patients completed the Swedish version of the SCQIPP questionnaire on the day of discharge or POD 5. Fifteen patients (29\%) asked the data collector to read the questions and complete the answers in the HAD scale preoperatively and five (10\%) received help to complete the questionnaires on discharge day or POD 5.

All the patients received the same preoperative information. What distinguishes the study from the general ward routine was the regular measuring of the VAS score, at rest and when coughing, during the hospital stay until discharge or POD 5. Prior to the study, the general ward routine was to discontinue documenting the VAS score after the chest tube had been removed.

On the day of the surgery, POD 0, the VAS scores were measured directly at the postanesthesia care unit (PACU), at rest and when coughing, by the ICU duty nurse, and on the general surgical ward by the registered nurse responsible for the patients. The duty nurse repeated the same procedure on the ward at 12 am and $6 \mathrm{pm}$. The VAS scores, patients' characteristics and pain treatment were documented in a specific study protocol. All members of the staff were informed about the study. The data collector conducted a pain assessment interview three respectively six months after the surgery via a telephone call. The questions were: Do you have any pain related to the lung surgery?. If yes: VAS score at rest and at worst/coughing.

\section{Data analysis}

The data was coded and analyzed using the SPSS version 20.0 (IBM Corp., Armonk, NY, USA). For descriptive purposes, continuous variables are presented as mean and standard deviation or median and $\mathrm{min} / \mathrm{max}$ where appropriate. Categorical data are presented as numbers and percentages. VAS and HAD are considered qualitative variables. The variables were examined using descriptive statistics and plots to assess normality, distribution and checking for outliners. For the analysis of the difference between groups, non-parametric tests were used, due to the small sample sizes and the not normally distributed groups. Mann-Whitney Utest (M-W) was used to test differences between two independent variables. Pearsons' Chisquare test was used to explore the relationships between every two independent categorical variables in this study. Wilcoxon signed-rank test was used to test the differences of paired values or percentages between two repeated measurements. The level of statistical significance was set at 0.05 . 


\section{Ethical considerations}

Ethical approval was obtained from The Ethics Committee of the University of Gothenburg, Sweden (code 245-11), following the Helsinki Declaration $^{21}$ regulations regarding human subjects research.

\section{Results}

Of the 52 patients invited, 51 had given informed consent to participate in this study. The main reasons for refusal of participation were fatigue and poor health. Characteristics of the participants are summarized in Table 1.

\section{Pain management and surgical procedure}

TEA was given to $44 / 51$ patients, $28 / 44$ were female. In 34/44, no TEA problems were found. Of identified TEA problems; dislocation was found in $6 / 10$, problem with the patient-controlled analgesia (PCA) pumps in $3 / 10$, and side effects in $1 / 10$. Those with dislocation problems received a new epidural catheter, those with pump problems received a new PCA pump, and a lower epidural infusion rate was prescribed where low blood pressure was reported. None of them were converted to IV analgesic. The reasons for not receiving TEA were: failure to insert the epidural catheter $4 / 7$, the catheter was inserted but did not function $1 / 7$, the patient had a medical condition which was contraindication for epidural catheter $1 / 7$, and the patient declined the treatment $1 / 7$. All the patients in the IV analgesic group were male and the surgical procedures were thoracotomy $6 / 7$ and VATS $1 / 7$. Length of surgery (LoS) and discharge day are found in Table 2.

\section{Pain intensity during hospital stay, mean and standard deviation (visual analogue scale: 0-100 $\mathrm{mm}$ )}

In the TEA group, the mean pain score at rest varied slightly. On POD $0-5$, the mean pain score was $<22 \mathrm{~mm}$, at each subsequent time. When coughing, the mean pain score at PACU was $24 \mathrm{~mm}$, subsequently increasing with a peak on POD 2 of $52 \mathrm{~mm}$, and thereafter ending on POD 5 with a mean pain score of $48 \mathrm{~mm}$.

In the IV analgesic group, the mean pain score at rest was $49 \mathrm{~mm}$ in PACU. On the general surgical ward during POD 0 , the mean pain score was $48 \mathrm{~mm}$, thereafter subsequently decreasing until POD 4 to $<15 \mathrm{~mm}$. The mean score when coughing, at PACU, was $49 \mathrm{~mm}$, subsequently increasing with a peak of $67 \mathrm{~mm}$ on POD 1, thereafter decreasing to $48 \mathrm{~mm}$ on POD 4. The mean pain score POD 0-4, in the two different pain treatment groups, is shown in Figure 1. The standard deviation of the experience of pain varied widely between participants.

Patients receiving IV analgesic recorded a significantly higher VAS score at rest, in PACU, $(\mathrm{P}=0.049 \mathrm{M}-\mathrm{W})$, and during $\mathrm{POD} 1$, at rest $(\mathrm{P}=0.004 \mathrm{M}-\mathrm{W})$, compared to the TEA group. No significant differences were found in the measured pain scores between women and men who received TEA. Younger patients, $<65$ years (median 65), experienced higher pain levels compared to the elderly patients, $\geq 65$ years, in the entire study group, and when coughing.

A comparison of pain scores and discharge day, $<5$ and $\geq 5$, (median 5), in the entire study group, revealed no significant differences. A comparison of $\mathrm{LOS}$ revealed a significantly higher pain score during POD 1-2, when LoS was $\geq 91$ min (median=91). This applied to the entire study group and the TEA group, revealing the pain score was significantly higher both at rest $(\mathrm{P}=0.018 \mathrm{M}-\mathrm{W})$ and when coughing $(\mathrm{P}=0.030 \mathrm{M}-\mathrm{W})$.

Mild (visual analogue scale: 0-30 $\mathrm{mm}$ ), moderate (visual analogue scale: $31-70 \mathrm{~mm}$ ) or severe pain (visual analogue scale: $71-100 \mathrm{~mm}$ ) A division of VAS into three categories shows that moderate pain was found with quite a linear distribution in a variation between $22-58 \%$ and severe pain with a variation between 11$26 \%$ during POD 0-5, when coughing, in the entire study group, as illustrated in Figure 2.

\section{Quality of pain management measured by the strategic and clinical quality indicators in postoperative pain management questionnaire assessment}

The quality of pain management assessment shows that the achieved total mean score in the SCQIPP questionnaire was a total of 61 out of 70 , a result that was not high enough to indicate an overall high quality of care, which required $>63$. However, high quality of care was found in individual questions concerning; the staff believed them when they told them about the pain, the staff cooperated well in treating their pain, they asked them every day to measure pain. Areas for improvement were found in subscale communication; the staffs' knowledge about the pain treatment they had received, action subscale; receiving help in finding a comfortable position in bed, and environment; there was no peace and quiet in the room at night. Measuring patients' satisfaction revealed that $88 \%$ of the patients were satisfied or very satisfied with the pain management. Those patients reporting more pain than expected were less satisfied with the quality of pain management.

Table 1. Patient characteristics.

\begin{tabular}{lcccc} 
& No. $(\%)$ & Mean, SD & Median & Min/Max \\
Age & 51 & $61.1,14.5$ & 65.0 & $18-82$ \\
Female & $28(54.9)$ & $62.0,10.5$ & 64.5 & $39-79$ \\
Male & $23(45.1)$ & $60.1,18.3$ & 65.0 & $18-82$ \\
\hline ASA * & & - & - & - \\
Class I-II & $30(58.8)$ & - & - & - \\
Class III & $21(41.2)$ & & & \\
\hline
\end{tabular}

SD, standard deviation. ${ }^{*} \mathrm{ASA}(\mathrm{I}-\mathrm{V})$ is a classification of anesthesia risk factors according to the American Society of Anesthesiology.

Table 2. Length of surgery and discharge day.

\begin{tabular}{lccccc} 
& No. & $\begin{array}{c}\text { Los }(\min ) \\
\text { Median }\end{array}$ & Min/Max & $\begin{array}{c}\text { Discharge day } \\
\text { Median }\end{array}$ & Min/Max \\
All surgery procedures & 51 & 91 & $16 / 271$ & 5 & $3 / 21$ \\
Thoracotomy & 44 & 91 & $16 / 271$ & 6 & $3 / 21$ \\
\hline VATS & 7 & 99 & $33 / 206$ & 4 & $3 / 6$ \\
Wedge resection & 20 & 57 & $16 / 155$ & 5 & $3 / 10$ \\
\hline Lobectomy & 27 & 120 & $40 / 271$ & 6 & $3 / 21$ \\
Pulmectomy & 4 & 80 & $41 / 95$ & 6 & $5 / 8$ \\
TEA & 44 & 89 & $16 / 271$ & 6 & $3 / 17$ \\
IV analgesic & 7 & 93 & $25 / 206$ & 5 & $3 / 21$ \\
\hline
\end{tabular}

LoS, length of surgery; VATS, video-assisted thoracic surgery; TEA, thoracic epidural analgesia. 
More pain than expected was found in $39 \%$ of the patients.

\section{Hospital anxiety and depression scale analysis}

No significant difference in anxiety or depression was found by comparing the HAD score pre- and postoperatively and also no significant correlation between the preoperative anxiety and the postoperative pain. A separate comparison of gender revealed that women had a significant reduction of anxiety postoperatively ( $\mathrm{P}=0.007$ Wilcoxon signed-rank test).

Pain three and six months, mild (0-30 mm), moderate (31-70 mm), severe $(71-100 \mathrm{~mm})$ pain

At the three month follow up, $76 \%$ experienced mild pain, $20 \%$ experienced moderate pain and $4 \%$ experienced severe pain, related to surgery. At the sixth month, 84\% experienced mild pain, $16 \%$ experienced moderate pain and no one experienced severe pain, in the entire study group. A significant reduction of pain from the third to the sixth month, $(\mathrm{P}=0.007$, using Wilcoxon signed-rank test), was found within the entire group (Figure 3).

At the three month follow up, $36 \%$ required analgesic for the pain and, at the six month follow up, $14 \%$ required analgesic for the pain. One of the patients required the opioid analgesic oxycodone after both three and six months.

\section{Discussion}

The clinical pain guideline with the gold standard VAS score of $<40 \mathrm{~mm}$ as the best practice, during the hospital stay, was achieved in this study when the pain score was measured at rest, but not when coughing. During POD 1-5, the mean pain score increased to VAS $40-50$ $\mathrm{mm}$, when coughing, regardless of pain treatment. Comparing the two different types of pain treatment, IV analgesic had significantly higher pain scores compared to the TEA group, during the first two postoperative days. This result is not unexpected from a clinical point of view within the hospital. However, due to the small sample size, this finding cannot be generalized to a larger population. The high pain score in the TEA group POD 0-2, when coughing, was not expected, as TEA is a well-documented superior treatment compared to other methods of pain management after lung surgery. ${ }^{9,7}$ There were no differences between the two groups on PODs 2-4, similar to a previous study performed in the same department, which compared TEA and IV analgesic after cardiac surgery. ${ }^{22}$ In that study, preoperative anxiety among the surgical patients was found to be associated with their levels of postopera-

\section{Mean VAS}

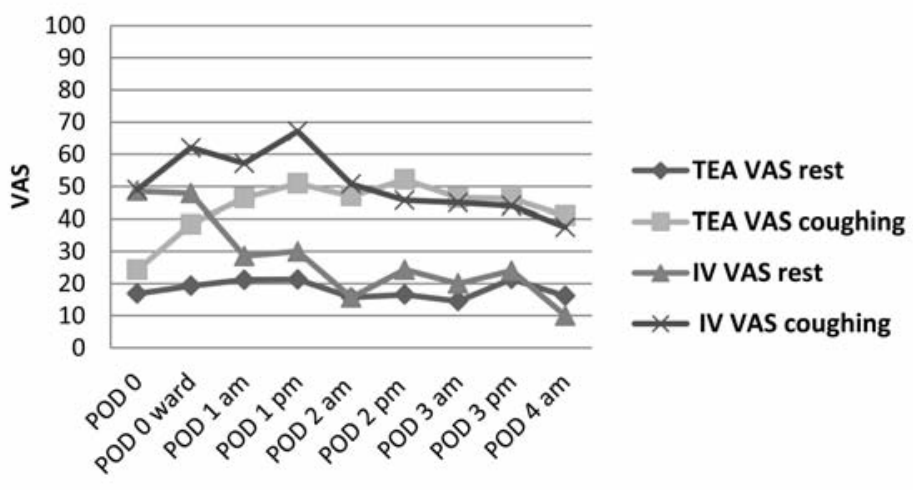

Figure 1. Mean visual analogue scale (VAS) score (0-100); thoracic epidural analgesia (TEA) and IV analgesic group, at rest and coughing, during post-operative day (POD) 0-4.

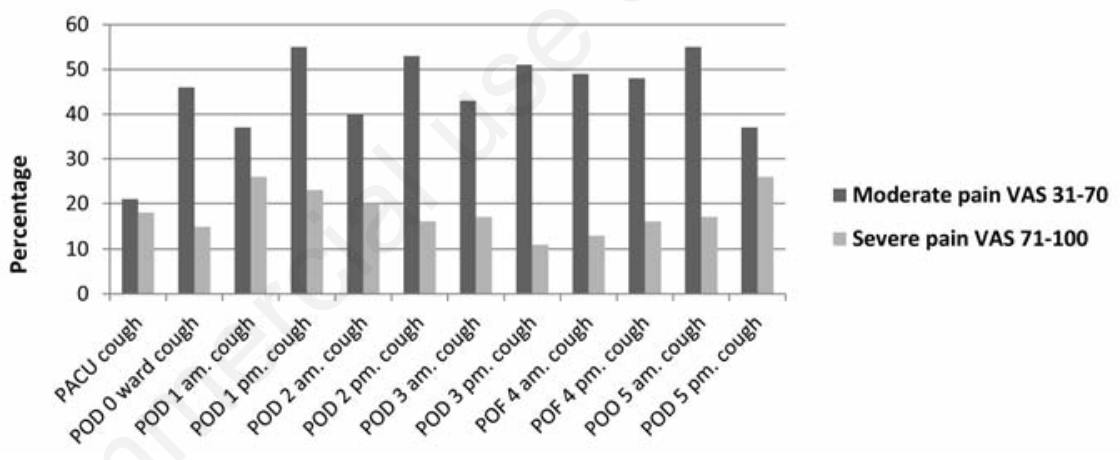

Figure 2. Percent of patients, in the total group, with moderate and severe pain, when coughing, during their hospital stay. Post-operative day (POD) 0-2, n=51; POD 3, n=47; POD 4, n=36; POD 5, n=25. VAS, visual analogue scale.

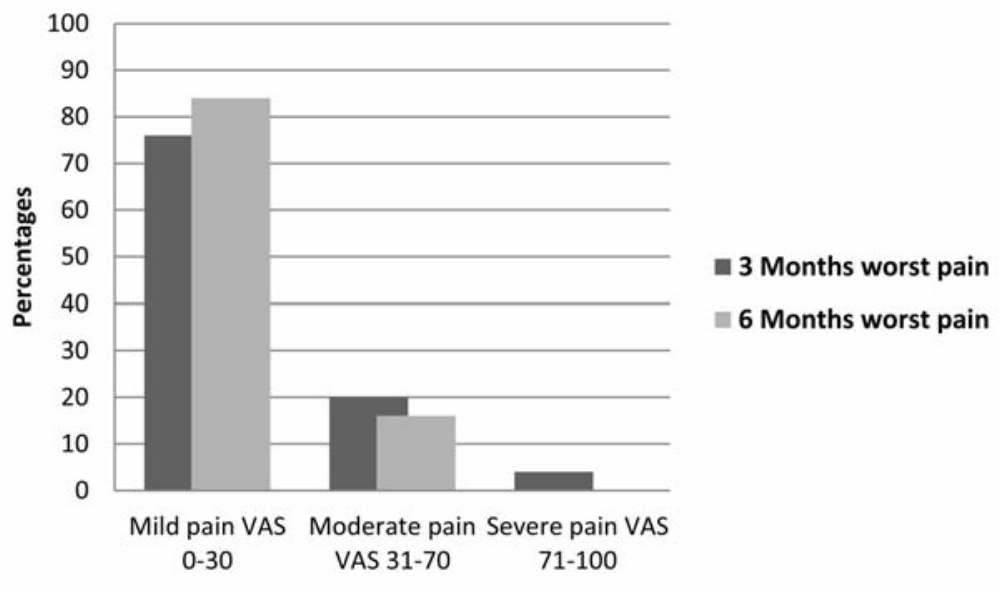

Figure 3. Percentages of patients with, mild, moderate or severe pain at three and six months, $n=49$. VAS, visual analogue scale. 
tive pain; ${ }^{12}$ however, this close relationship was not found in this study.

The findings that younger patients, aged $<65$ years, had more pain than older patients during the hospital stay and those with a longer surgery time, more than $90 \mathrm{~min}$, also experienced more pain than patients with a shorter surgery time (e.g., on the first postoperative day only), were consistent with our previous understanding about the predictive factors of postoperative pain. ${ }^{1}$

The results in this study are consistent with others that report moderate and severe pain after surgery and insufficient pain management, despite available multimodal analgesia. ${ }^{23,24}$ Tocher et al. ${ }^{24}$ found that almost $70 \%$ of the patients experienced pain during their hospital stay, $26 \%$ of the patients had pain all or most of the time and $12 \%$ had severe pain. Lorentzen et $a .^{23}$ reported an incidence of moderate pain in $51 \%$ of the patients and $36 \%$ experienced severe pain postoperatively.

In this study, the total SCQIPP score did not achieve the desired score for high quality of care in the postoperative pain management. Furthermore, it revealed areas for improvement in all of the subscales. Previous studies where the SCQIPP instrument has been used show both similar and divergent results. In this sample, high quality was achieved for the question the staff believed in the patients' pain, which had also been found earlier. ${ }^{19} \mathrm{~A}$ high quality result for the question the staff cooperated well in treating my pain was also achieved. ${ }^{19}$ This is an important component in optimal pain management.

The study results state that $88 \%$ of the participants were either satisfied or very satisfied with the postoperative pain management and less satisfaction was found with higher pain levels. This corresponds with results from other studies. ${ }^{19,23,25}$ Tocher et al. ${ }^{24}$ found that patients with severe pain reported less satisfaction with the pain management. However, a study from Germany shows that $55 \%$ of the patients who had undergone surgery were dissatisfied with their pain management. ${ }^{26}$ Svensson et al. ${ }^{25}$ found that most of the patients, 91\%, expected moderate and severe pain after surgery, when questioned prior to surgery. When questioned postoperatively, $76 \%$ of the patients experienced such pain levels. Interestingly, $81 \%$ were satisfied with the pain management levels. Idvall et al. ${ }^{27}$ found that patients seemed more satisfied with the postoperative pain management than the nurses assessed them to be.

In this study, $39 \%$ of the patients' had more pain than expected, which seems to be a high incidence compared to other studies using the same questionnaire. For example, Gunningberg and Idvall ${ }^{19}$ reported that $20 \%$ of the patients at a department of thoracic surgery, both cardiac and lung surgery, had more pain than expected and 15\% reported more pain than expected at a department of general surgery. Idvall et al. ${ }^{17}$ found that $24 \%$ of the patients reported more pain than expected. The patients in both studies assessed the quality of care lower, which is similar to this study.

Stomberg and Öman ${ }^{28}$ discuss the importance of having a realistic expectation of postoperative pain, maintaining that nurses play a vital role in informing the patients before surgery, to promote a realistic expectation of postoperative pain. The discrepancy between expectation and satisfaction is an interesting issue and quite complex. It demonstrates the complexity of evaluating postoperative pain management and measuring satisfaction with pain management alone is not recommended. ${ }^{25}$

The experienced pain after three and six months in this study is difficult to compare with other studies, due to the lack of a unanimous definition of persistent/chronic post-surgical pain. It is however a well-known problem and explains the variations in the reported incidence of chronic pain..$^{29,5}$ In this sample, $16 \%$ experienced moderate pain after six months and no one experienced severe pain, which is slightly under the documented incidence of chronic pain after lung surgery. ${ }^{1,2}$ The different surgical techniques might affect the pain experience of individual patients. Recent studies have indicated fewer respiratory complications and shorter lengths of hospital stay after VATS, when compared to thoracotomy. ${ }^{30}$ However, there is no consensus regarding whether the VATS technique can reduce the incidence of postoperative or chronic pain, although it has been shown to reduce severe persistent pain. ${ }^{30}$

\section{Conclusions}

A large number of patients experienced moderate and severe pain postoperatively and more than one-third had more severe pain than expected. However, most of the patients were satisfied with the quality of pain management received. The findings confirmed the severity of pain experienced after lung surgery and demonstrated an apparent need for continued improvement in postoperative pain management after lung surgery. Moderate pain still existed among $16 \%$ of the patients 6 months after surgery. Nevertheless, the sample size in this study was small and only from one hospital, thus reducing the generalization of the findings. Another weakness is the absence of power analysis.

It is important to note that pain should be assessed not only at rest but also in movement. In addition, patients' preoperative expectations of postoperative pain should be addressed.

\section{References}

1. Kehlet H, Jensen TS, Woolf CJ. Persistent postsurgical pain: risk factors and prevention. Lancet 2006;367:1618-25.

2. Macrae WA. Chronic post-surgical pain: 10 years on. Br J Anaesth 2008;101:77-86.

3. Ochroch EA, Gottschalk A, Augostides J, et al. Long-term pain and activity during recovery from major thoracotomy using thoracic epidural analgesia. Anesthesiology 2002;97:1234-44.

4. Breivik H, Collett B, Ventafridda V, et al. Survey of chronic pain in Europe: prevalence, impact on daily life, and treatment. Eur J Pain 2006;10:287-333.

5. Wildgaard K, Ravn J, Nikolajsen L, et al. Consequences of persistent pain after lung cancer surgery: a nationwide questionnaire study. Acta Anaesthesiol Scand 2011;55:60-8.

6. Gottschalk A, Cohen SP, Yang S, Ochroch EA. Preventing and treating pain after thoracic surgery. Anesthesiology 2006;104: 594-600.

7. PROSPECT. Procedure specific postoperative pain management; 2007. Available from: http://www.postoppain.org Accessed: November 2013.

8. Davies R, Myles P, Graham J. A comparison of the analgesic efficacy and side-effects of paravertebral vs epidural blockade for thoracotomy - a systematic review and metaanalysis of randomized trials. Br J Anaesth 2006;96:418-26.

9. De Cosmo G, Aceto P, Gualtieri E, Congedo E. Analgesia in thoracic surgery: review. Minerva Anestesiol 2009;75:393-400.

10. Steegers MAH, Snik DM, Verhagen AF, et al. Only half of the chronic pain after thoracic surgery shows a neuropathic component. J Pain 2008;9:955-61.

11. Wickström Ene K. Postoperative pain management: predictors, barriers and outcome. Göteborg: Institute of Health and Care Sciences, The Sahlgrenska Academy at the University of Gothenburg; 2008.

12. Jeantieu M, Gaillat F, Antonini F, et al. Postoperative pain and subsequent ptsdrelated symptoms in patients undergoing lung resection for suspected cancer. J Thorac Oncol 2014;9:362-9.

13. McNamara MC, Harmon D, Saunders J. Effect of education on knowledge, skills and attitudes around pain. Br J Nurs 2012; 21:958-64.

14. Wilson B. Nurses' knowledge of pain. J Clin Nurs 2007;16:1012-20.

15. Dihle A, Bjølseth G, Helseth S. The gap between saying and doing in postoperative pain management. J Clin Nurs 2006; 15:469-79.

16. Hunter S. Determination of moral negli- 
gence in the context of the undermedication of pain by nurses. Nursing Ethics 2000;7:379-91.

17. Idvall E, Hamrin E, Sjöström B, Unosson M. Patient and nurse assessment of quality of care in postoperative pain management. Qual Saf Health Care 2002;11:327-34.

18. Idvall E, Berg A. Patient assessment of postoperative pain management orthopaedic patients compared to other surgical patients. ONA J 2008;12:35-40.

19. Gunningberg L, Idvall E. The quality of postoperative pain management from the perspectives of patients, nurses and patient records. J Nurs Manag 2007;15:756-66.

20. Zigmond AS, Snaith RP. The hospital anxiety and depression scale. Acta Psychiatr Scand 1983;67:361-70.

21. Helsinki WD. [Ethical principles for medical research involving human subjects Etiska riktlinjer för omvårdnad i Norden]; 2000. Available from: http://www.codex.vr.se
Accessed: November 2013. [In Swedish].

22. Hansdottir V, Philip J, Olsen MF, et al.

Thoracic epidural versus intravenous patient-controlled analgesia after cardiac surgery: A randomized controlled trial on length of hospital stay and patient-perceived quality of recovery. Anesthesiology 2006; 104:142-51.

23. Lorentzen V, Hermansen IL, Botti M. A prospective analysis of pain experience, beliefs and attitudes, and pain management of a cohort of Danish surgical patients. Eur J Pain 2012;16:278-88.

24. Tocher J, Rodgers S, Smith MAC, et al. Pain management and satisfaction in postsurgical patients. J Clin Nurs 2012;21: 3361-71.

25. Svensson I, Sjöström B, Haljamäe H. Influence of expectations and actual pain experiences on satisfaction with postoperative pain management. Eur J Pain 2001 ;5:125-33.
26. Maier C, Nestler N, Richter H, et al. The quality of pain management in German hospitals. Dtsch Arztebl Int 2010;107:607.

27. Idvall E, Berg K, Unosson M, Brudin L. Differences between nurse and patient assessments on postoperative pain management in two hospitals. J Eval Clin Pract 2005;11:444-51.

28. Stomberg Warrén M, Öman UB. Patients undergoing total hip arthroplasty: a perioperative pain experience. J Clin Nurs 2006;15:451-8.

29. Niraj G, Rowbotham D. Persistent postoperative pain: where are we now? Br J Anaesth 2011;107:25-9.

30. Scott WJ, Allen MS, Darling G, et al. Videoassisted thoracic surgery versus open lobectomy for lung cancer: a secondary analysis of data from the American College of Surgeons Oncology Group Z0030 randomized clinical trial. J Thorac Cardiovasc Surg 2010;139:976-83. 